\title{
A fibre Bragg grating based inclinometer system for ground movement measurement
}

\author{
Jin Li, Ricardo Correia, Edmond Chehura, Stephen Staines, Stephen W. James* and Ralph P. Tatam \\ Engineering Photonics Group, School of Engineering, Cranfield University, \\ Bedfordshire, MK43 0AL, UK
}

\begin{abstract}
The measurement of ground movement is an essential part of many geotechnical engineering operations. For decades, inclinometer systems have traditionally been used for this purpose to provide crucial information to engineers and researchers. However, conventional inclinometer systems have their limitations, such as high cost and poor durability. In this paper a fibre optic based inclinometer system is reported, which utilizes fibre Bragg grating sensors attached to the casing of a conventional inclinometer. The characterization of the sensor revealed good agreement with theory and conventional displacement measurements. For a casing of length $2.5 \mathrm{~m}$, the minimum measureable deflection of the top of the casing was found to be $0.48 \mathrm{~mm}$ when the FBGs were interrogated with a $1 \mathrm{pm}$ wavelength resolution system.
\end{abstract}

Keywords: Ground movement measurement, inclinometer system, fibre Bragg grating

\section{INTRODUCTION}

The measurement of ground movement is an essential part of many geotechnical engineering operations. Inclinometer systems have been used widely to monitor ground movements in various applications, for example, landslides, tunnels, and foundations, etc., where they provide vital ground movement information including magnitude, rate and location. The data produced can be used to verify design assumptions and provide early warning of problems ${ }^{1}$.

A conventional inclinometer system consists of a plastic casing that is installed in a near vertical position in the ground, with a servo-accelerometer or electro-level sensor inserted into the casing to measure the local tilt of the casing in response to ground movement. The sensor element is lowered and raised, guided by grooves in the inner surface of the casing, with the tilt of the casing being recorded at fixed spatial intervals. In case an automated system is needed, a series of sensors can be placed in the casing with the assistance of appropriate wheel sets. The deflection profile is then measured along the whole length of the casing, which complies with the ground movement around it $^{1}$. However, these inclinometer systems have limitations including, for example, high cost, poor durability and reduced accuracy operating under steeply inclined conditions

Over the past decade, fiber optic sensors, in particular fibre Bragg grating (FBG) sensors have gained popularity in ground movement measurement in geotechnical engineering applications, e.g., slopes ${ }^{2}$, braced excavations ${ }^{3}$, and tunnels using single core ${ }^{4}$ as well as multi core fibre ${ }^{5}$. Fibre optic sensors offer a number of generic advantages, including immunity to electromagnetic interference, flexibility and durability and small dimensions. In addition to their small dimensions, FBGs offer the ability to multiplex a number of sensors in a single optical fibre, and can operate over long distances with no downlead sensitivity.

In this paper, a simple and effective FBG based inclinometer system is presented, which operated based on the beam theory and the FBG's inherent strain sensing feature. The inclinometer system was fabricated and tested in the laboratory with high accuracy and resolution results obtained.

\section{SENSING PRINCIPLE}

A pair of FBG arrays is attached onto the surface of a flexible sensing tube diametrically opposite to each other with FBGs orientated in parallel with the neutral axis of the tube at various positions, as schematically illustrated in Figure 1, where subscripts "a" and "b" donate FBG arrays a and b, respectively. If the tube is deflected in the plane of the two FBG arrays with a direction as indicated by the arrow, the FBGs in array a are tensioned whilst the FBGs in array $b$ are compressed (e.g., $\varepsilon_{i \mathrm{a}}$ and $\varepsilon_{i \mathrm{~b}}$ in Figure 1). According to the elastic beam theory ${ }^{6}$, the deflection at a point $x$ along the length of the tube can be determined from the strain profile by the following equation

Fourth European Workshop on Optical Fibre Sensors, edited by José Luís Santos, Brian Culshaw,

José Miguel López-Higuera, William N. MacPherson, Proc. of SPIE Vol. 7653, 765314

(C) 2010 SPIE · CCC code: 0277-786X/10/\$18 - doi: 10.1117/12.866334

Proc. of SPIE Vol. 7653 765314-1 


$$
v(x)=\frac{1}{r} \iint \varepsilon(x) d x d x
$$

Where $v$ is the deflection of the tube, $r$ is the distance to the neutral axis, in this case is outer radius of the tube, and $\varepsilon$ is strain profile and determined as such: The strain at measurement point $i$ is defined as

$$
\varepsilon_{i}=\frac{\left(\varepsilon_{i \mathrm{a}}-\varepsilon_{i \mathrm{~b}}\right)}{2}
$$

The strain profile along the entire length of the tube is then deduced by applying curve fitting to the discrete measured strain data. It is also worth noting that temperature would affect both FBG arrays equally, as the configuration is symmetric. According to equation 2 , the effect can favorably be canceled out, and thus making the sensor insensitive to the temperature variations. This self thermally compensated feature has also been demonstrated experimentally, for example, by $\mathrm{Xu}$ et $\mathrm{al}^{7}$.

In case that the predominant deflection direction cannot be anticipated, a biaxial version can be made to suit for general 2D conditions, where two pairs of FBG arrays are needed with each pair being attached onto the tube surface orthogonal to each other. The tube is then inserted in ground either in drill holes or embedded in retaining structures. Due to deformation compatibility, the tube responds to the movement of the surrounding ground.

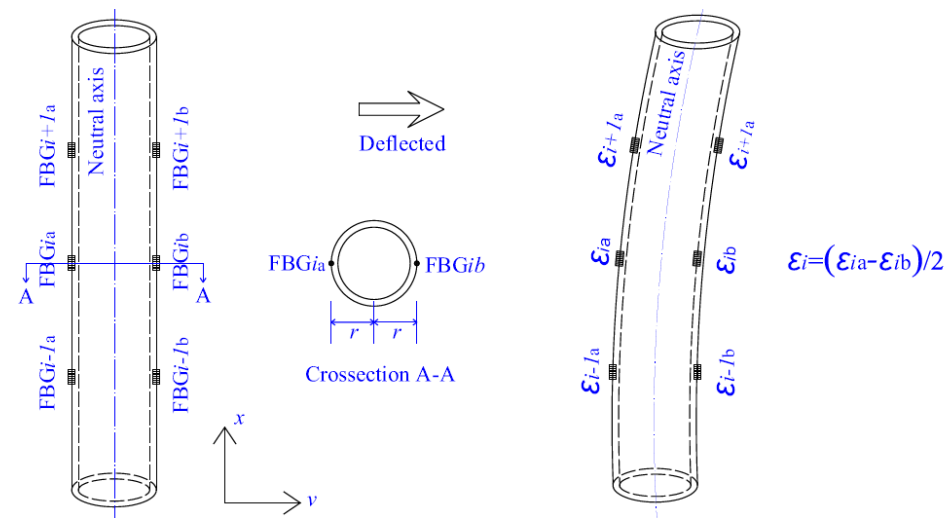

Figure 1 Working principle of a FBG based inclinometer system

\section{SENSOR FABRICATION}

The inclinometer system developed in this study comprises a conventional inclinometer casing, two FBG arrays, fibre patchcords and connectors. A 3m long and 70mm OD Easy Connect inclinometer casing (Soil Instruments Limited) was used as the sensing tube. It is a specially manufactured ABS extrusion with precise grooves formed at $90^{\circ}$ into the internal surface. This is particularly beneficial to field tests, as it also permits a conventional inclinometer probe to be used independently for verification purpose.

Two FBG fibre arrays were fabricated to a typical reflectivity of $40 \%$ by a holographic side-writing technique in single mode photosensitive fibres (Fiberscope, PS1250). The protective plastic coating was removed before irradiating the fibre. Each array consisted of six wavelength division multiplexed FBGs with Bragg wavelengths centred at $1526 \mathrm{~nm}$. The length of each FBG was $5 \mathrm{~mm}$, and the physical spacing between adjacent FBGs was $0.5 \mathrm{~m}$. The FBGs, which were not recoated after fabrication, were bonded onto the casing's outer surface, diametrically opposite each other, using a cyanoacrylate adhesive, which had been demonstrated adequate by some of the authors in previous work, making measurements on a n ABS pipe ${ }^{8}$. Each FBG array was oriented such that it was aligned with the central line of one of the guide grooves of the inclinometer casing. The physical location of the sensors is shown in Figure 2.

The FBG arrays were then protected by covering the entire length of each fibre with an epoxy resin, and terminated with fibre patchcord and FC connector ends. The robustness of this packaging technique has proven to be effective when the authors have deployed FBGs sensors in pile testing applications ${ }^{10}$. This configuration, in addition, allows many to be joined both physically and optically with little time in the field to the required depth of interest. 


\section{LABORATORY PROVING TESTS}

The performance of the inclinometer system in the measurement of deflection was characterised in the laboratory. The system was set up on the laboratory bench as a cantilever beam with fibre Bragg grating arrays aligned in the plane of the induced deflection. Due to limitations introduced by the length of the bench, deflection was applied manually, in a direction transverse to the axis of the inclinometer casing, at a location $2.468 \mathrm{~m}$ from the fixed end by a translation stage. The deflections at the FBG positions were also measured by dial gauges with a resolution of $0.0127 \mathrm{~mm}$. A schematic diagram illustrating the FBG positions along the cantilever beam and a photograph of the experiment set up are provided in Figures 2 and 3, respectively.

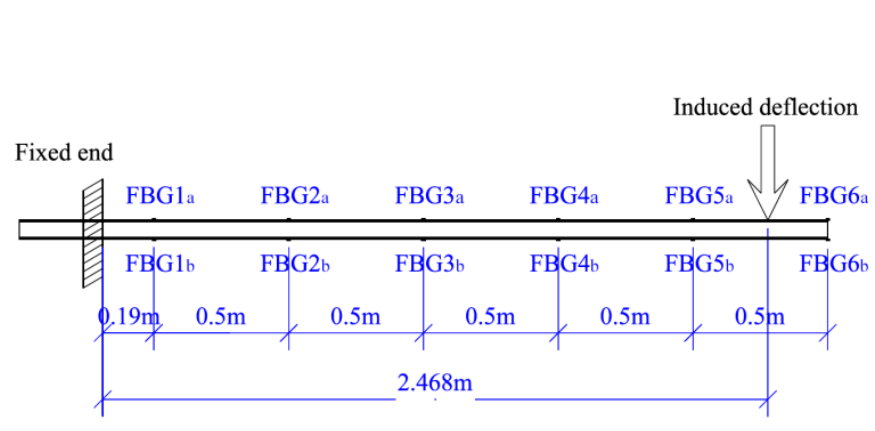

Figure 2 Details of the cantilever beam

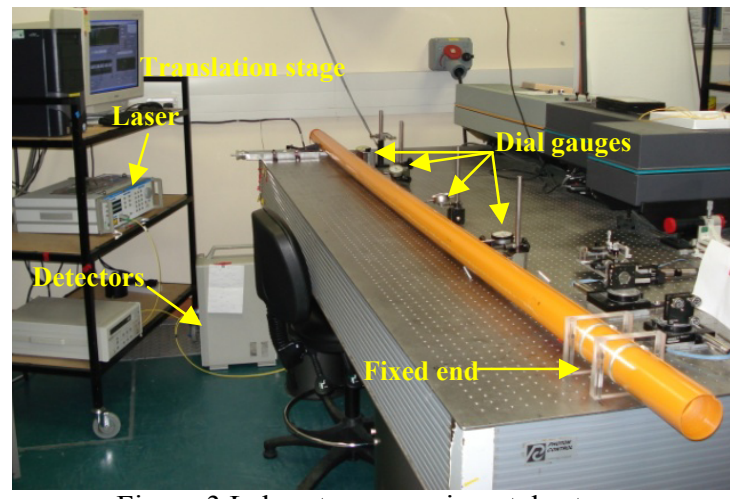

Figure 3 Laboratory experimental set up

A tunable laser (Tunics-plus) was used to monitor the Bragg reflection spectrum. The Bragg wavelengths of the two FBG arrays were determined with reference to an NIST-traceable gas cell (RSM 2514). A wavelength resolution of 1 $\mathrm{pm}$, corresponding to a strain resolution of $0.845 \mu \varepsilon$, can be attained with this system. The schematic of the senor integration system is shown in Figure 4.

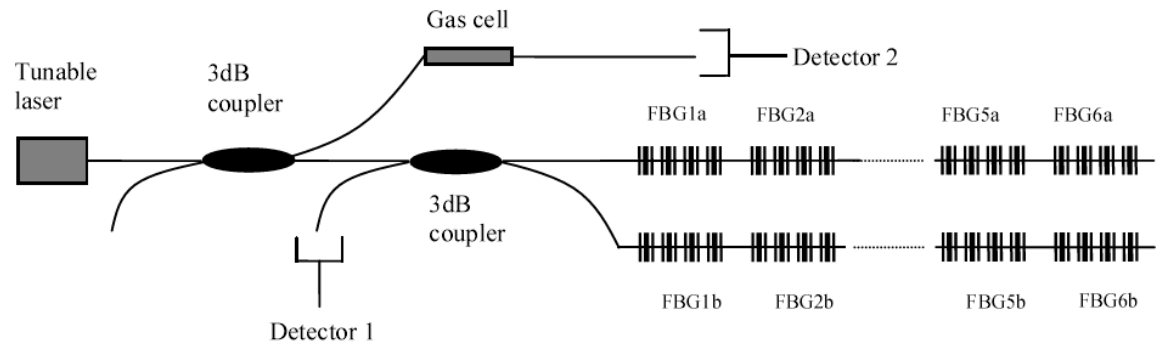

Figure 4 Schematic of the sensor interrogation system

Typical strains and deflections at FBG positions along the casing are presented in Figures 5 and 6, along with the theoretical values and dial gauge measurements. The theoretical values were calculated using the model of a known displacement at the free end of cantilever beam ${ }^{7}$. The FBG derived deflections at FBG positions were determined using equation 1.

Figure 5 shows that the FBG strain measurements were in good agreement with the theory and that the derived deflections also agreed well with both theory and dial gauge measurements (Figure 6). The slight difference between FBG and dial gauge could be attributed to alignment and positioning errors when attaching the FBG arrays onto the casing. The difference between the measurements and theory showed that the discrepancy of the laboratory setup from a perfect cantilever given the high precision nature of the measurement. For a casing of length $2.5 \mathrm{~m}$, the minimum measureable deflection of the top of the casing was found to be $0.48 \mathrm{~mm}$ when the FBGs were interrogated with a $1 \mathrm{pm}$ wavelength resolution system. Comparing with commercially available inclinometers which can resolve the range from 2 to $6 \mathrm{~mm}$ for a $30 \mathrm{~m}$ measurement ${ }^{1}$, the performance of this FBG based inclinometer system is considered sufficient for the most practical applications 


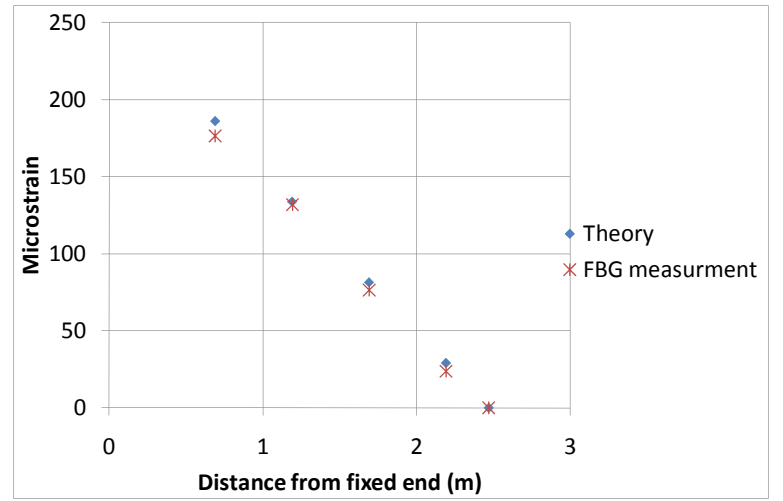

Figure 5 Typical strain at FBG positions

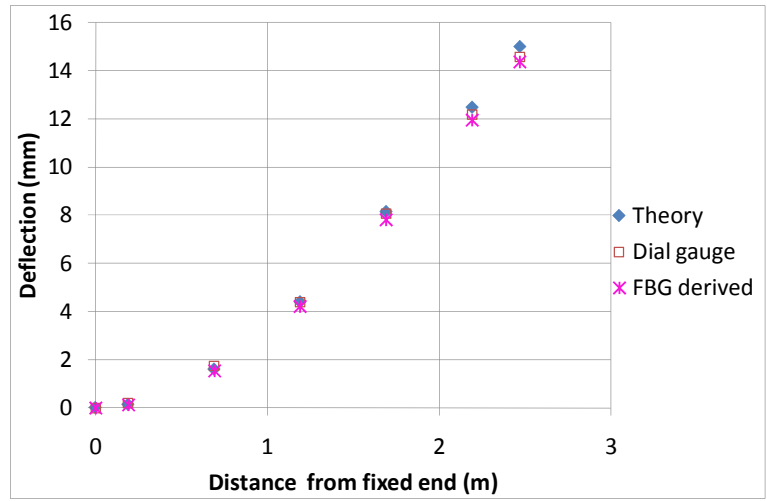

Figure 6 Typical deflection at FBG positions

A field trial, either a slope or foundation project, will be undertaken to further validate the performance of the inclinometer system. Resolution can further be increased by using a larger diameter casing, if necessary. In addition, this also increases the stiffness of the system thus making it possible to make the measurement depth longer. Particularly, it could operate under larger ground movement condition, whereas conventional sensors compromise substantially their accuracy.

\section{CONCLUSIONS}

A fibre optic based inclinometer was developed by using fibre Bragg gratings attached to a conventional inclinometer casing. Laboratory based characterisation showed it to be capable of measuring deflection accurately, with a resolution sufficient for the most practical applications, with good agreement with theory and conventional displacement measurement techniques.

\section{ACKNOWLEDGEMENT}

This research project was funded by the Engineering and Physical Sciences Research Council (Grant No. EP/D506654/1).

\section{REFERENCES}

[1] Dunnicliff, J., [Geotechnical instrumentation for monitoring field performance], John Wiley \& Sons Inc, New York, (1993).

[2] Yoshida, Y., Kashiwai, Y., Murakami, E., Ishida, S. and Hashiguchi, N., "Development of the monitoring system for slope deformations with fiber Bragg grating arrays,” Proc. SPIE 4694, 296-303 (2002).

[3] Ho, Y., Huang, A. and Lee, J., "Development of a fibre Bragg grating sensored ground movement monitoring system," Meas. Sci. Technol. 17, 1733-1740 (2006).

[4] Metje, N., Chapman, D., N., Rogers, C., D., F., Henderson, P. and Beth, M., "An optical fiber sensor system for remote displacement monitoring of structures - Prototype tests in the laboratory," Struct. Health Monitoring 7(1), 51-63 (2008).

[5] MacPherson, W., N., Silva-Lopez, M., Barton1, J., S., Moore1, A., J., Jones, J., D., C., Zhao, D., Zhang, L., Bennion, I., Metje, N., Chapman, D., N. and Rogers, D., D., F., "Tunnel monitoring using multicore fibre displacement sensor," Meas. Sci. Technol. 17, 1180-1185 (2006).

[6] Gere, J. M. and Timoshenko, S. P., [Mechanics of Materials], Nelson Thornes, Cheltenham, (2002).

[7] Xu, M., Archambault, J., L., Reekie, L. and Dakin, J., P., "Thermally-compensated bending gauge using surfacemounted fibre gratings," Intl Journal of Optoelectronics 9(3), 281-283 1994).

[8] Groves, R., Chehura, E., Li, W., Staines, S., E., James, S., W. and Tatam, R., P., "Surface strain measurement: a comparison of speckle shearing interferometry and optical fibre Bragg gratings with resistance foil strain gauges," Meas. Sci. Technol. 17, 1175-1184 (2007).

[9] Li, J., Correia, R., Chehura, E., Staines, S., James, S., W., Tatam, R., P., Butcher, A. P. and Fuentes, R., "Field monitoring of static, dynamic, and statnamic pile loading tests using fibre Bragg grating strain sensors," Proc. SPIE 7503,750340 (2009). 